\section{DEL 68 AL 15M: CONTINUIDADES Y RUPTURAS ENTRE CICLOS DE PROTESTA}

Eduardo Romanos

Universidad Complutense de Madrid ORCID iD: https://orcid.org/0000-0002-0200-3470 eromanos@ucm.es

Cómo citar este artículo/Citation: Romanos, E. (2018). Del 68 al 15M: continuidades y rupturas entre ciclos de protesta. Arbor, 194 (787): a430. https://doi.org/10.3989/arbor.2018.787n1003

Recibido: 31 enero 2017. Aceptado: 13 noviembre 2017

RESUMEN: La protesta no se distribuye aleatoriamente en el tiempo, sino que se concentra en forma de ciclos. Los últimos tres grandes ciclos internacionales de protesta han sido los surgidos alrededor del 68, el movimiento antiglobalización en el cambio de milenio y la reciente movilización por la democracia y contra la austeridad a partir de 2010. Este artículo analiza las continuidades entre estos ciclos centrándose en el impacto cultural de los movimientos sociales a partir de la elaboración y difusión de nuevos conceptos de democracia.

PALABRAS CLAVE: movimientos sociales; movimiento antiglobalización; protestas anti-austeridad; democratización; difusión de innovaciones.

\section{FROM 1968 TO THE INDIGNADOS: CONTINUITIES AND DISCONTINUITIES BETWEEN CYCLES OF PROTEST}

Copyright: (C) 2018 CSIC. Este es un artículo de acceso abierto distribuido bajo los términos de la licencia de uso y distribución Creative Commons Reconocimiento 4.0 Internacional (CC BY 4.0).

ABSTRACT: Protest is not randomly distributed over time but concentrates in the form of cycles. The last three major international cycles of protest have been those surrounding 1968 , the global justice movement at the turn of the millennium and the recent mobilization for democracy and against austerity from 2010. This article analyzes the continuities between these protest cycles, focusing on the cultural impact of social movements through the development and diffusion of new concepts of democracy.

KEYWORDS: social movements; global justice movement; anti-austerity protest; democratization process; diffusion of innovations. 


\section{INTRODUCCIÓN}

La protesta no se distribuye aleatoriamente en el tiempo sino que se concentra en determinados periodos históricos. Son los Ilamados ciclos de protesta, que van dejando un poso en relación con nuevas formas de confrontación pero también con nuevas estrategias para hacerles frente por parte de los estados (el control policial de la protesta) y, de una manera más general, con el desarrollo mismo de la democracia. Si nos centramos en los movimientos sociales, veremos que estos no tienen que inventar la rueda a cada rato (della Porta, 2017). Las innovaciones que trae consigo cada ciclo de protesta en el terreno de la acción y la organización de los movimientos sociales se van depositando en forma de repertorios, esto es, "el conjunto de medios que tiene [un grupo] para plantear reivindicaciones diferentes a individuos diferentes" (Tilly, 1986, p. 2. Citado en della Porta y Diani, 2011, p. 218). Aunque la difusión de innovaciones se acelere en el inicio de los ciclos de protesta (Tarrow, 1994), los nuevos desarrollos beben en realidad del trabajo cultural realizado por redes sumergidas de activistas en periodos menos visibles pero igualmente importantes. Son lo que Alberto Melucci (1994) Ilamó periodos de latencia, en los que el conflicto está más silenciado pero no apagado del todo. Estos periodos se alternan con otros de (mayor) visibilidad en el desarrollo habitual de los movimientos sociales.

Este artículo analiza las continuidades y discontinuidades entre los últimos ciclos de protesta más importantes a nivel internacional, a saber, los surgidos alrededor del 68, el movimiento antiglobalización en el cambio de milenio y la reciente movilización por la democracia y contra la austeridad a partir de 2010, para ver qué queda de uno en otro. El artículo se estructura como sigue: los tres primeros apartados examinan por separado cada uno de los ciclos mientras que el cuatro se centra en su impacto cultural a partir de un análisis longitudinal de la experimentación con nuevos conceptos de democracia. Un último apartado de conclusiones cierra el artículo subrayando los principales resultados de la investigación.

\section{LA HETEROGENEIDAD DEL 68 GLOBAL}

Protestas masivas se habían sucedido desde principios de la década de 1960 contra el rearme nuclear, la guerra de Vietnam, el Shah de Irán o el golpe de estado en 1967 de los coroneles en Grecia, pero ninguna de ellas tuvo la repercusión del 68 francés (Mazower, 2000, p. 318). En pocos días, una huelga general masiva paralizó la economía, mientras los estudiantes ocupaban las universidades, colapsando el sistema educativo. La protesta universitaria tuvo como foco el nuevo y marginal campus de Nanterre, fundado cuatro años antes en el cinturón rojo de $\mathrm{Pa}$ rís para descongestionar el primigenio de la Sorbona. Algunos de sus estudiantes formaron el movimiento 22 de marzo que ocupó en esa fecha la universidad en protesta por la detención dos días antes de varios compañeros acusados de romper una ventana del edificio de American Express en una manifestación contra la guerra de Vietnam (Ross, 2002, p. 90). Tras varias jornadas de disturbios con la policía, el rectorado decidió cerrar el campus de Nanterre el 2 de mayo, trasladándose la protesta a la Sorbona y desde allí por toda la ciudad y otras localidades de Francia (Seidman, 2004, pp. 92 y ss.)

Luc Boltanski (2002, p. 7) ha señalado cómo en mayo de 1968 se unieron dos formas de crítica: la social, enarbolada por el movimiento obrero contra la desigualdad, la pobreza y la explotación, y una crítica artística relacionada con el romanticismo revolucionario y ocupada en desvelar "la arbitraria subestructura de un orden sin fundamentos". La síntesis entre ambas, obra del estudiantado, abriría según Boltanski un nuevo ciclo político. Frente a esta imagen del 68 como cesura, Gilles Lipovetsky (1994) ha subrayado su carácter clausurante. Los acontecimientos de mayo habrían significado "la glorificación del espíritu neolibertario" y el fin de los conceptos modernos de la revolución y el individuo, elementos de la ideología revolucionaria "guiada por una fe escatológica y un partido todopoderoso", el comunista, hegemónico entre la izquierda hasta ese momento. A partir de entonces, emergería un nuevo individualismo, que Lipotvesky denomina transpolítico, implosionador de las dicotomías modernas: "lo político y lo existencial, lo público y privado, lo ideológico y lo poético, la lucha colectiva y la gratificación personal, la revolución y el humor, todo devino entrelazado de manera inextricable".

Sin embargo, esta identificación del fenómeno del 68 como acontecimiento extraordinario que separa un antes y un después parece pasar por alto el proceso de gestación de las demandas que se hicieron visibles en la movilización de aquellas jornadas. Sería quizás más acertado pensarlo, de acuerdo al modelo bipolar de Melucci (1994), como expresión de cambios producidos en el activismo revolucionario durante las últimas décadas, sobre todo en el anarquismo, que remozó alguno de sus principios clásicos con nuevas inquietudes y preocupaciones (Romanos, 2007). En Francia, los existencialistas habían erosionado el concepto de- 
cimonónico de naturaleza humana y su vinculación con la necesidad, esto es, con el camino trazado hacia su satisfacción a través de la revolución social, clausurando así su carácter teleológico (Crowder, 1991; Morland, 1997; Yack, 1986). Defendían un socialismo con más voluntad y menos determinación al mismo tiempo que advertían sobre los peligros del afán destructivo y la violencia (véase Aron, 1973). A uno y otro lado del Atlántico, había surgido una 'tercera vía' que quiso renovar la izquierda denunciando simultáneamente la tiranía del totalitarismo comunista y la subyugación individual y colectiva de los regímenes democráticos capitalistas (Katsiaficas, 1987). En 1968, la juventud se rebelaba contra las coerciones impuestas desde los sistemas políticos y educativos proponiendo una transformación de la vida cotidiana que acabara con la futilidad de su existencia, al mismo tiempo que descubría los caminos de la emancipación social en una autoliberación de la persona (Kellner, 2005; Sanbonmatsu, 2004). En este sentido, los anteriores movimientos de provos y situacionistas habían contribuido a afianzar una crítica social que abría las limitaciones del orden establecido mediante gestos revolucionarios que mostraban su debilidad y la capacidad creadora del individuo (De Jong, 1971; McDonough, 2002; Pas, 2008).

Más allá del contexto francés, donde la movilización, aunque intensa, duró poco (en las elecciones de junio el partido de de Gaulle salió reforzado), el 68 fue una compleja crisis global con múltiples ramificaciones. Europa formaba ya de por sí un microcosmos, dividido en dos bloques, una miríada de tradiciones políticas y un amplio espectro de oportunidades políticas (Klimke y Scharloth, 2008). Allí, acontecimientos internacionales de consecuencias transnacionales, como la guerra de Vietnam o la primavera de Praga, tuvieron un impacto de similar magnitud en activistas a uno y otro lado del telón de acero, mientras eran interpretados de manera diferente entre círculos activistas de un mismo país. Trabajadores manuales ayudaron a canalizar la protesta hacia una solución política en Francia mientras que hicieron de catalizador antiparlamentario en Italia. En algunos países, las protestas surgieron a partir de acontecimientos dramáticos (como el asesinato de Benno Ohnesorg en Berlín occidental) mientras que en otros no hicieron falta esos "puntos de inflexión icónicos" (Nehring, 2008, p. 130). En ocasiones, culturas políticas tradicionales desempeñaron un papel paradójico como facilitador de ideologías más jóvenes, como el puritanismo con las demandas maoístas por la simplicidad y la autenticidad en Noruega y Suecia. La dimensión lingüística fue motivo de conflicto en Bélgica mientras que barre- ras lingüísticas debilitaron las protestas en Suiza. Las iglesias locales desempeñaron papeles diferentes en diferentes países: inhibiendo la protesta en Rumanía, aliándose con la disidencia en Polonia o colaborando circunstancialmente con los disidentes en España. La diversidad y complejidad del ciclo de protesta se refleja también en los diferentes factores que explican el final de las movilizaciones: la represión policial (Suiza e Irlanda del Norte), la intervención militar exterior (Checoslovaquia) o la interferencia de demandas nacionalistas (Yugoslavia), entre otros.

A pesar de estas diferencias, podemos decir que mayo del 68 significó en su conjunto una revitalización y actualización de principios antiautoritarios vinculados con la tradición de pensamiento libertario y, lo que quizás es más importante, la irrupción de la imaginación en la protesta, no solo en sus repertorios de acción, sino también en sus principios, fines y metas. Si el "corto verano" de 1936 fue la apoteosis del anarquismo en el terreno de la acción y de las realizaciones revolucionarias (Enzensberger, 1972), la corta pero intensa primavera de 1968 condensó el trabajo cultural que desde el final de la segunda guerra mundial se venía produciendo en diversas "comunidades críticas" (Rochon, 1998) y movimientos de protesta.

\section{UN MOVIMIENTO DE MOVIMIENTOS (PREEXISTENTES)}

Los ciclos de protesta se caracterizan no solo por un gran número de acciones (concentradas en un breve periodo de tiempo) sino por un salto cualitativo en sus formas. Como sostiene Tarrow (1994), los ciclos de protesta expanden las formas conocidas de acción colectiva (repertorios) a partir de pequeñas innovaciones. La innovación en las formas de confrontación se acelera en los periodos de intensificación de los conflictos. Las protestas del ciclo alrededor del 68 incluían pequeñas innovaciones asociadas con tácticas de no-violencia, desobediencia civil y teatro callejero que pasaron a formar parte del repertorio de acción colectiva empleado por los movimientos sociales (Kornetis, 2013). Teach-ins organizadas por los estudiantes para subvertir la verticalidad universitaria, détournements, happenings y otras formas antiritualistas de acción directa tenían, más allá de una función instrumental, también una dimensión expresiva, orientada a la transformación de los propios activistas, concienciándoles del carácter represivo de sus sociedades (Klimke y Scharloth, 2008, p. 5).

Más allá de los acontecimientos de protestas, los turbulentos años 60 vieron el surgimiento de los llamados "nuevos movimientos sociales", unos movimientos que 
giraban "en torno a cuestiones como los derechos de las mujeres, las relaciones de género, la preservación medioambiental, la etnicidad y la emigración, la paz y la solidaridad internacionales, con una fuerte (y nueva) base social de clase media y una clara diferencia respecto de los modelos de acción colectiva obrera o nacionalista que les habían precedido en la historia" (della Porta y Diani, 2011, p. 15). Durante la década de 1970 , estos nuevos movimientos sociales mantuvieron un perfil confrontacional relativamente alto (Ilegando incluso a formas radicales de acción) mientras que en la década siguiente muchos de ellos acabarían por moderarse e institucionalizarse, cristalizando en estructuras fuertemente profesionalizadas. La moderación en las formas de acción colectiva corrió pareja en muchos países a una moderación, no sin pausas ni retrocesos, en las estrategias de control policial de la protesta. Se pasó de un modelo de escalada de fuerza a uno de control negociado caracterizado por una reducción del uso de la fuerza, un mayor énfasis en el "diálogo" y la inversión de grandes recursos en la recopilación de información (della Porta y Reiter, 1998).

Hasta que llegó el movimiento antiglobalización. La conocida como "batalla de Seattle" protagonizada a finales de noviembre de 1999 por la policía de esa ciudad, con el apoyo de la guardia nacional y otras fuerzas especiales, y activistas que se manifestaban en contra de la Ronda del Milenio organizada por la Organización Mundial del Comercio supuso un punto de inflexión en la visibilidad de un naciente movimiento transnacional contra la globalización neoliberal, y de la incapacidad de las fuerzas del orden para hacerle frente. Después de Seattle, los activistas antiglobalización continuaron organizando contra-cumbres y acciones de protesta ante las grandes reuniones internacionales: el Foro Económico Mundial de Davos, las cumbres europeas o el G8. También crearon su propio foro de encuentro, el Foro Social Mundial, cuya primera edición se celebró en la ciudad brasileña de Porto Alegre en 2001. Por su parte, la policía siguió rearmándose, llegando incluso a la militarización (en términos de equipamiento, entrenamiento y despliegue) de unidades dedicadas al control del orden público, y empleando una estrategia fuertemente coercitiva, de empleo muchas veces indiscriminado de la fuerza, en combinación con estrategias de recopilación dirigida de información como medida preventiva (della Porta y Diani, 2011, pp. 253-255).

El movimiento por una justicia global, o movimiento antiglobalización (aunque en realidad dirigía su crítica contra una versión particular de la misma: la globa- lización neoliberal), se ha entendido muchas veces como un "movimiento de movimientos" preexistentes que se reactivaron gracias al esfuerzo de muchos activistas por establecer puentes entre sus diferentes marcos de referencia (frame bridging). La identidad del movimiento se fue forjando en el diseño y el desarrollo de eventos transnacionales (campañas, contracumbres, foros), que requerían la participación de organizaciones creadas en la resaca de la anterior ola de movilización en torno a cuestiones muy diversas. Se fueron creando así densas redes de interacción entre estas organizaciones. Los activistas dejaban a un lado sus lealtades particulares para participar en amplias movilizaciones que servían de sustrato sobre el que construir la identidad inclusiva del nuevo movimiento.

Donatella della Porta y Lorenzo Mosca (2007) han definido este proceso como "contaminación en acción". Generado a través de la participación individual y organizativa en campañas comunes, la contaminación en acción implica diversos mecanismos con funciones diferentes: estructural (facilitando la coordinación logística entre las organizaciones), afectiva (generando la tolerancia y la confianza mutua entre los activistas) y cognitiva (favoreciendo la construcción de identidades intersectoriales y transnacionales). Della Porta y Mosca analizan este proceso en dos sectores aparentemente enfrentados y con dinámicas y valores opuestos: sindicatos y grupos promotores de la solidaridad con el sur global. Se ha identificado a los primeros con valores materiales, el egoísmo y la defensa de sus propios intereses, mientras los segundos lo harían más bien con valores postmateriales, el altruismo y la solidaridad. Unos y otros convergieron en un movimiento que supuso la revitalización de la "cuestión social" al mismo tiempo que mostraba una fuerte preocupación por muchas de las demandas centrales en los nuevos movimientos sociales surgidos alrededor del 68 en temas relacionados con los derechos de las mujeres y las minorías, la desigual distribución de los riesgos medioambientales o la amenaza de la guerra. Si el 68, según Boltanski (2002), fusionó dos formas de crítica (social y artística), el nuevo ciclo de protesta alrededor del cambio de milenio combinó dos conjuntos de demandas hasta entonces fuertemente divididos (materialistas y postmaterialistas).

\section{UN NUEVO CICLO DE INDIGNACIÓN}

El ciclo de protesta vinculado con el movimiento antiglobalización decreció a comienzos del nuevo milenio, en parte acelerado por su crisis en Estados Unidos después del 11-S en un ambiente significativamente 
más represivo, con la vinculación que desde el poder se hacía entre activismo transnacional y terrorismo global y la dominación del problema de la guerra por encima de otras demandas (Hadden y Tarrow, 2007). Sin embargo, el siguiente ciclo no tardó en llegar. Lo hizo muy pronto esta vez. La década de 2010 se abría con una fuerte oleada de movilización en diferentes regiones del mundo sobre cuestiones de alguna forma interrelacionadas: desde las políticas de austeridad, con una fuerte crítica al poder de las multinacionales y la creciente desigualdad, hasta la defensa de la democracia, con movilizaciones contra regímenes autoritarios y reivindicaciones a favor de una "democracia real ya". Ancladas en contextos muy diferentes, las movilizaciones dentro de esta nueva ola presentan sin embargo algunos rasgos en común, desde las emociones (con la indignación en el centro) y formas de acción (de ocupación del espacio público) hasta marcos rectores (que vinculan la crisis financiera a una crisis de la democracia) e identidades colectivas, pasando por infraestructuras (de redes y reuniones) y experiencias generacionales (de precariedad y acceso a las nuevas tecnologías de la información) (Castells, 2012; Flesher Fominaya, 2014; Gerbaudo, 2012; Glasius y Pleyers, 2013; della Porta y Mattoni, 2014; Tejerina, Perugorria, Beenski y Langman, 2013).

El reciente ciclo de protesta encontró en España uno de sus focos principales. El 15 de mayo de 2011, la convocatoria de manifestaciones en todo el país con el slogan "No somos mercancía en manos de políticos y banqueros: idemocracia real ya!" significó el pistoletazo de salida de un movimiento cuya capacidad de movilización y visibilidad no tienen parangón en la historia reciente de España (Romanos, 2013a). Algunos de los indignados que se movilizaron contra la respuesta de las autoridades a la crisis económica participaron más tarde en la creación de nuevos partidos políticos que hoy en día están gobernando algunas ciudades importantes y se han hecho un hueco en los parlamentos, tanto a nivel regional como nacional. Mientras tanto, un fuerte ciclo de movilización ha tenido lugar. Miles de personas han participado en un movimiento de protesta que ha intentado, y en buena medida conseguido, cambiar la responsabilidad de la crisis del nivel individual (de la gente que "vivió por encima de sus posibilidades") al colectivo (de las elites políticas y económicas, coaligadas para defender sus intereses). Los activistas han exigido a las autoridades que revirtieran los recortes en servicios públicos y derechos civiles, fortalecieran mecanismos de control y transparencia en su gestión y crearan nuevos canales de acceso a la toma de decisiones. Sin embargo, las autoridades han hecho en buena medida oídos sordos, amparadas por unas instituciones diseñadas durante la transición a la democracia para alejarlas de la presión de la calle. De hecho, las respuestas más contundentes han sido negativas, en la forma de leyes "mordaza" que extienden el rango de acciones colectivas susceptibles de ser sancionadas.

A lo largo del nuevo ciclo de protesta, los indignados han organizado un amplio rango de acciones, unas más convencionales, otras más contundentes, con una gran capacidad de movilización y masivo apoyo social (Romanos, 2014; Sampedro y Lobera, 2014). Las movilizaciones han provocado un cambio en el campo doméstico de los movimientos sociales con el surgimiento de nuevos actores (por ejemplo, asambleas locales, iniciativas colectivas de autogestión, las llamadas "mareas" en diferentes sectores de empleo y servicios públicos) y el fortalecimiento de otros ya existentes (entre otros, la Plataforma de Afectados por la Hipoteca y el movimiento de paralización de desahucios). En sus protestas y redes, los activistas han enfrentado la lógica del sistema con una lógica alternativa basada en una versión actualizada del modelo de democracia deliberativa (véase la siguiente sección). Se han afanado por construir un movimiento de 'cualquiera' basado en un 'nosotros' terriblemente inclusivo que pretende superar viejas filias ideológicas o partidistas y las dinámicas autoreferenciales, formas organizativas, discursos e identidades de movimientos sociales tradicionales (Perugorria y Tejerina, 2018).

A su vez, como ya se ha adelantado, el movimiento $15 \mathrm{M}$ se inserta en una oleada de protesta más amplia que tiene en la Primavera Árabe y el movimiento Occupy Wall Street otras de sus manifestaciones más visibles. Participantes en estas movilizaciones se han vinculado unos con otros, no solo en la identificación de una misma lucha en pos de mayores cotas de democracia, sino también a través de la difusión de prácticas e ideas (Romanos, 2016). Sin embargo, muchas de estas prácticas e ideas no son nuevas. Investigadores en movimientos sociales han establecido importantes líneas de continuidad entre la nueva ola de protesta transnacional y la protagonizada por el movimiento antiglobalización. De alguna forma el movimiento antiglobalización adelantó la reciente crisis económica (y política) como consecuencia de un proceso de globalización dirigido por y en aras del mercado. Como señalan Donatella deIla Porta y Alice Mattoni (2014), este movimiento lanzó una llamada de atención, una alerta sobre lo que, si no se ponían cortapisas a la globalización neoliberal, se avecinaba sobre los grupos más vulnerables de la(s) 
sociedad(es). La nueva ola de protestas anti-austeridad tiene como protagonista a estos grupos, que han experimentado ya esas consecuencias en el contexto de la actual crisis económica y financiera. Los indignados de hoy vinculan los efectos negativos del capitalismo global neoliberal con una noción ineficaz e insuficiente de democracia (Flesher Fominaya, 2014), como lo hacían los activistas diez años antes. Y frente a esa versión ineficiente de la democracia ellos plantean otro modelo, más participativo y deliberativo, con el que experimentaron de forma visible en las plazas.

Sin embargo, no todo son continuidades entre las dos olas de movilización. Una de las diferencias más importantes tiene que ver con el locus de la crítica y la acción. Si a lo largo de la historia los movimientos sociales han encontrado en el estado-nación su principal objetivo y ámbito de actuación, los activistas antiglobalización se desplazaron hacia un nivel más transnacional. Las protestas se organizaron principalmente en ese nivel y a ese nivel pertenecían también los responsables de la versión neoliberal de la globalización que ellos criticaban: organizaciones como el Banco Mundial, el Fondo Monetario Internacional o la Organización Mundial del Comercio. Estas organizaciones siguen siendo criticadas por quienes se manifiestan hoy contra las políticas de austeridad, pero la actual ola de protesta ha invertido de alguna forma la tendencia para anclarse de nuevo en el territorio. De hecho, una de las formas más visibles de acción ha sido el establecimiento de campamentos más o menos estables en los centros simbólicos de poder local y nacional. Por ejemplo, los indignados han elegido las plazas centrales como lugar en el que representar su denuncia contra la mercantilización de la vida social y el espacio público.

Esa denuncia no se ha detenido. Al contrario, se ha hecho todavía más fuerte contra intervenciones del mercado todavía más autoritarias en protestas como la de Gezi (Turquía), que desvelan el autoritarismo de las actuales formas de la globalización neoliberal. En 2013 la mercantilización del espacio público en Estambul (con el intento de construir un centro comercial que iba a sepultar uno de los pocos parques en el centro de la ciudad) encontró una fuerte resistencia que desbordó la protesta inicial para dar vida a un nuevo movimiento social dentro de una nueva fase en la secuencia global de movilizaciones. Justo cuando la ola transnacional que se hizo más visible en 2011 parecía decaer, protestas como la de Turquía, Brasil y otras latitudes renovaron el enfrentamiento contra el capitalismo global neoliberal y sus consecuencias (della Porta, 2017).

\section{EL HILO DEMOCRÁTICO ENTRE LOS CICLOS DE PROTESTA $^{1}$}

Uno de los objetivos principales de los movimientos sociales ha sido el desarrollo de nuevos conceptos de democracia (della Porta y Diani, 2011, p. 239). Los movimientos reclaman la legitimidad de modelos alternativos a la democracia parlamentaria. Por lo general no piden la abolición del actual sistema de partidos, sino que buscan dotarle de un mayor contenido democrático al mismo tiempo que se involucran en la generación de espacios alternativos en los que experimentar con otros modelos de democracia. En ese plano parecen actuar también los recientes movimientos contra la austeridad, y en concreto el $15 \mathrm{M}$, donde el grueso de los participantes presentaba un perfil más bien reformista (Calvo, 2013), por mucho que las reformas que promovían se orientasen hacia cambios generales en la forma en que se organiza y conduce la política.

Los nuevos movimientos sociales desarrollados predominantemente en Europa alrededor del 68 invocaron una teoría democrática comunitarista que podemos vincular con la tradición anarquista. La democracia directa se erigió en alternativa a los modelos por entonces dominantes con una concepción más elitista y competitiva: el modelo liberal de democracia y la democracia organizada de corte socialista o católico (Kitschelt, 1993). Si el 68 global trajo consigo la actualización del concepto de democracia directa, la siguiente ola de movilización transnacional por una globalización desde abajo puso en práctica un nuevo concepto de democracia deliberativa.

Donatella della Porta (2005a, p. 340) ha definido la democracia deliberativa como aquella que se da cuando, "bajo unas condiciones de igualdad, inclusividad y transparencia, un proceso comunicativo basado en la razón (la fuerza de un buen argumento) es capaz de transformar las preferencias individuales y alcanzar decisiones orientadas al bien público". El movimiento antiglobalización experimentó con este modelo alternativo de democracia en sus redes internas. Los activistas participaron de una "cultura organizativa" que "enfatizaba la diversidad por encima de la homogeneidad; la subjetividad por encima de la obediencia a las demandas organizativas; la transparencia incluso a costa de la efectividad; la confrontación abierta orientada a la construcción del consenso por encima de la toma de decisiones eficiente; y la 'contaminación ideológica' por encima del dogmatismo" (della Porta, 2005b, p. 73). Esta cultura se regía asimismo por un marcado sentido de prefiguración. Los activistas en- 
tendían que la mejor estrategia para traer consigo el cambio social que ellos anhelaban era practicar ese cambio en el seno de su propio movimiento (MaeckeIbergh, 2011). Sin embargo, la estrategia no es nueva. Su origen vincula de nuevo a los nuevos movimientos sociales con la tradición del movimiento anarquista. Aunque parezca una innovación sesentayochista, la prefiguración es tan vieja como la teoría anarquista moderna: Bakunin y sus seguidores abogaban por una sociedad sin clases que, lejos de tomarse por asalto, debía ser construida a imagen y semejanza de la Asociación Internacional de los Trabajadores. La Internacional era el "embrión" de ese nuevo mundo. De la misma manera, los proyectos alternativos de los anarquistas en otras esferas, diferentes aunque relacionadas con el asociacionismo obrero, tales como la educación, la cultura y la información, también debían servir de modelo para la sociedad anti-autoritaria, libre y no-jerarquizada que perseguían sus organizaciones, grupos y activistas (Romanos, 2013b).

¿Hasta qué punto el nuevo ciclo internacional de protesta iniciado en 2010 se hace eco de este modelo de democracia? Un vistazo a sus prácticas y discursos parece mostrar un profundo compromiso con el mismo. Centrando el foco de nuevo en España, y concretamente en las primeras fases del movimiento 15M, el marco organizativo adoptado durante las acampadas fue el asambleario. La horizontalidad asamblearia fue el mecanismo de decisión del incipiente movimiento. Quienes participaban en las asambleas se sentían iguales y parte de un proyecto compartido. En su desarrollo se hacía hincapié en la necesidad de utilizar un lenguaje inclusivo, una de cuyas expresiones más visibles era el empleo del género femenino (en referencia a "personas"). De hecho, la inclusividad ("absoluta, de cualquier persona") era el fundamento para mantener la subjetividad y la diversidad dentro del movimiento. La transparencia no fue solo una demanda hacia sus principales adversarios (en el sistema político y el económico) sino también una de los valores centrales del movimiento: seguramente no hay mejor forma de visibilizar el proceso deliberativo e invitar a participar en él que celebrándolo en las plazas de los barrios, pueblos y ciudades. Además, las redes internas (comisiones y grupos de trabajo) hacían pública toda la información relativa a sus acuerdos y actas a través de Internet, facilitando su acceso a toda persona interesada en conocer sus actividades.

En cualquier caso, los principios de igualdad, inclusividad y transparencia son propios también del modelo de democracia directa y participativa desplegado por los nuevos movimientos sociales post-68. El valor añadido del modelo asociado al movimiento antiglobalización residiría en la importancia de la transformación de preferencias facilitada en el proceso deliberativo. De hecho, la deliberación es el modo de interacción más favorable para dicha transformación (Landwehr, 2009). Los teóricos de la democracia deliberativa rebaten en este punto el enfoque de elección racional propio del modelo liberal, que entiende el proceso democrático básicamente como una agregación de preferencias individuales (Talpin, 2011). Los teóricos liberales entienden que dichas preferencias deben protegerse de la perniciosa injerencia del estado. Los teóricos deliberativos critican este enfoque por su esencialismo, reduccionismo y problematicidad. En concreto, tratan de desmontar tres ilusiones asociadas al modelo liberal, subrayando que: 1) las preferencias no son autónomas ni libres, sino que se producen en determinados contextos económicos, sociales y culturales; 2 ) las preferencias pueden cambiar si lo hacen estos contextos; y 3) la agregación de preferencias supuestamente prefijadas de acuerdo a intereses individuales obstaculiza la promoción de la justicia social. En resumen, si cada uno votamos según nuestras preferencias individuales y estas están determinadas por intereses individuales, la toma de decisiones colectivas no puede orientarse al bien común. En cambio, la democracia deliberativa entiende que las preferencias son endógenas y maleables, siendo el objetivo de la deliberación su eventual transformación en aras del bien común a través del acceso a la información y un proceso consciente de aprendizaje (Talpin, 2011, p. 16).

El movimiento $15 \mathrm{M}$ se hacía eco del principio de transformación de las preferencias en la reflexión de sus activistas acerca del "pensamiento colectivo" y la "escucha activa" que debían guiar la participación en las asambleas:

El pensamiento colectivo es totalmente opuesto al sistema actual que se rige por un pensamiento individualista [...] Normalmente ante una decisión dos personas con ideas opuestas tenderán a enfrentarse y defender ferozmente sus ideas poniendo como objetivo convencer, ganar o a lo sumo llegar a un punto medio. El objetivo del pensamiento colectivo es construir. Es decir, dos personas con ideas diferentes ponen sus energías en construir algo. No se trata entonces de mi idea o la tuya. Son las dos ideas juntas las que darán un producto nuevo que a priori no conocíamos ni tú ni yo. Por eso es tan necesaria la escucha activa en la que no solo estamos preparando la réplica que vamos 
a dar. El pensamiento colectivo nace cuando entendemos que todas las opiniones, las nuestras y las diferentes, todas, son necesarias para generar la idea de consenso. Una idea que tras su construcción de forma indirecta nos transforma [...] [El pensamiento colectivo es] algo así como el resultado de la síntesis de las inteligencias e ideas individuales, no una sumatoria ecléctica, sino la síntesis. Las inteligencias individuales puestas al servicio del bien común, la creación a partir de la diferencia, entender la diferencia como un elemento que aporta enriquecimiento de la idea común (Guía rápida para la dinamización de asambleas populares publicada por acampadasol, 31/05/2011, disponible en http://madrid.tomalaplaza.net).

La transformación de preferencias en el proceso deliberativo debe estar sustentada en una comunicación de calidad. El debate y la deliberación, entendida como una forma "desapasionada, razonada y lógica" de comunicación (Dryzek, 2000, p. 64), permite la formación de nuevas preferencias orientadas al bien común. En este sentido, los activistas del 15M explicaban en ese momento la participación en la toma de decisiones orientada al consenso en las asambleas contraponiendo la "escucha activa" a lo que ocurre en los procesos de democracia representativa:

Se buscan los mejores argumentos para tomar la decisión más acorde con las diferentes opiniones, no posicionamientos enfrentados, como ocurre cuando se vota. Su desarrollo debe ser pacífico, respetando todas las opiniones, hay que dejar los prejuicios y las ideologías en casa [...] Es importante mantener calma gestual para no transmitir a la asamblea sentimientos o afectos personales, recordaremos en todo momento el valor de una sonrisa en momentos de tensión o bloqueo (Guía rápida para la dinamización de asambleas populares publicada por acampadasol, 31/05/2011, disponible en http://madrid.tomalaplaza.net).

Para velar por la calidad de la comunicación y facilitar otras condiciones del proceso deliberativo, las acampadas contaban con una comisión dedicada a la "dinamización de asambleas". Entre sus miembros había jóvenes profesionales formados en metodologías participativas (Nez y Ganuza, 2012, p. 123) que asumían el papel de moderadores y otros "facilitadores" que intentaban asegurar su concentración e imparcialidad. La comisión también elaboró y difundió diversos materiales escritos y audiovisuales que buscaban "ayudar a materializar esta nueva forma de hacer política", instruyendo en el proceso deliberativo. Se especificaban allí los diferentes actores, los espacios, las relaciones y los mecanismos del "modelo de es- tructura asamblearia y toma de decisiones", describiendo, por ejemplo, los roles de los ya mencionados moderadores y facilitadores, pero también las funciones de los equipos encargados de la preparación del espacio y las herramientas físicas (megafonía, sillas para personas con movilidad limitada, etc.), los turnos de palabra, los intérpretes de la lengua de signos y la redacción de las actas.

A pesar de estos esfuerzos por asegurar el buen funcionamiento del modelo deliberativo, su puesta en marcha no estuvo exenta de limitaciones y problemas. "La combinación de una voluntad de consenso con la deliberación abierta significa que la toma de decisiones puede ser fácilmente saboteada por grupos pequeños [...] Como quedó patente en las conversaciones con los activistas [del 15M], la toma de decisiones era lenta, laboriosa y desmoralizante cuando incluso las decisiones simples podrían ser bloqueadas por un puñado de personas que ejercen el poder de veto" (Feenstra, Tormey, Casero-Ripollés y Keane, 2016, p. 10). En cualquier caso, la experimentación con el modelo deliberativo practicado en las asambleas ha dejado una huella biográfica importante en los participantes con consecuencias en su modo de entender la política (Feenstra et al., 2016).

Las protestas del nuevo ciclo reproducen en buena medida la cultura organizativa y los nuevos conceptos de democracia gestados en anteriores ciclos de protesta. Sin embargo, las nuevas movilizaciones trajeron consigo pequeñas innovaciones, afianzadas en periodos menos visibles pero no por ello huérfanos de acción, sobre todo en la dimensión más simbólica (Romanos, 2013a). Estas innovaciones vinculaban ese trabajo cultural de anteriores periodos de latencia (Melucci, 1994) con las nuevas formas de protesta practicadas en el nuevo ciclo de movilización. En este sentido, podemos advertir dos aspectos de la inclusividad que resultan hasta cierto punto novedosos en el movimiento $15 \mathrm{M}$; aspectos que a su vez fueron difundidos a otros movimientos. "En primer lugar, la inclusividad promovida por los indignados no tiene tanto que ver con los ya participantes en el movimiento $-\mathrm{y}$ el establecimiento de mecanismos que aseguren su inclusión en el proceso de toma de decisiones- sino con los potenciales participantes. Aquí la plaza desempeñó un papel importante" (Romanos, 2016, p. 111). Se habían ocupado muchos espacios en el pasado pero no tanto el centro de las ciudades de manera masiva y con vocación de cierta permanencia (el 'modelo Tahrir' de protesta ${ }^{2}$ ). La novedad del 15M consistía en colocar la experimentación con un nuevo concepto 
de democracia en el centro del espacio público. Los activistas sacaban el "experimento" de la democracia deliberativa de los recintos que hasta entonces habían sido su "laboratorio" (centros sociales, foros sociales, etc.) para colocarlo en el centro de las ciudades, animando a "cualquiera" que pasara por allí a unirse y desbordar con ello los límites de la movilización. El cambio era en realidad un cambio de orientación: la atención se ponía en los potenciales participantes y no tanto en los ya comprometidos con el movimiento (Lawrence, 2013).

El segundo aspecto tiene que ver con un significado menos racional y más afectivo de la inclusividad, no tan orientado hacia el proceso de toma de decisiones (donde, como hemos visto, no es bueno traer "sentimientos o afectos personales") sino hacia la transformación del espacio público como un espacio abierto también a la empatía (Romanos, 2016). Los activistas entendían que uno de los valores centrales del movimiento era la "INCLUSIVIDAD. La fuerza de este movimiento es ser muchos y distintos [...] Los espacios que nos dan fuerza, que nos alegran y potencian, son los que permiten a cada cual sentirlos como propios". Este concepto de inclusividad, que podemos llamar inclusividad basada en la empatía, o inclusividad empática, dejaba a un lado las cuestiones más estratégicas para crear un espacio donde cualquiera pudiera compartir sus problemas favoreciendo con ello la apertura hacia personas sin una experiencia previa de participación y por ello más reticentes a involucrarse en el nuevo movimiento.

\section{CONCLUSIONES}

'1968' fueron en realidad varios años de contienda política y social, les années 68 (Ginsborg, Passerini, Strath y Wagner, 2002), que en perspectiva internacional podemos entender como un ciclo de protesta que contenía muchos conflictos. En diferentes partes del mundo, la juventud se rebelaba contra las coerciones impuestas desde el establishment político y educativo en pos de una transformación de la vida cotidiana que acabara con la futilidad de su existencia, al mismo tiempo que descubría los caminos de la emancipación social en una autoliberación de la persona. Uno de los resultados más visibles del ciclo de protesta fue, de hecho, la difusión de la autogestión como un modelo de organización en el mundo del trabajo y en formas alternativas de convivencia en el ámbito más privado. Los nuevos conflictos venían expresados en términos y conceptos avanzados por existencialistas e intelectuales adscritos o cercanos a la llamada new left. La protesta empleó además un repertorio de acción en buena medida renovado bajo la influencia de provos, situacionistas y otros movimientos contraculturales. Los activistas se organizaron siguiendo pautas no jerárquicas, horizontales y descentralizadas, en abierto contraste con el modo de estructuración vertical de los partidos políticos (Kornetis, 2013), y configurando una serie de formas que serían predominantes en los movimientos sociales a partir de entonces.

Las recientes protestas contra la austeridad y por una democracia "real" surgidas en diferentes regiones del mundo a partir de 2010 se vinculan con aquellas de '1968' a partir de ese repertorio de acción y organización que actúa como repositorio de los saberes de los movimientos sociales y, al mismo tiempo, horizonte de posibilidad que se va ampliando poco a poco con pequeñas innovaciones. Pero el vínculo se establece también a partir del desarrollo en las protestas recientes de conceptos de democracia participativa experimentados por los sesentayochistas, con el ciclo antiglobalización actuando de puente, sobre todo en relación con la dimensión más deliberativa de ese concepto. A su vez, como todo ciclo de protesta, el reciente ha traído innovaciones, que en este caso combinan formas de acción y democracia: los indignados sacaron la experimentación con la democracia deliberativa al centro de las ciudades, donde instalaron acampadas en señal de protesta. En las acampadas practicaban un sentido de inclusividad relativamente novedoso, fuertemente orientado al $99 \%$ de la población y a convertir el espacio público en un espacio abierto a la empatía.

Visto así, el trabajo de los movimientos sociales en las últimas décadas ha sido un esfuerzo orientado en buena medida a la ampliación de nuestro concepto de democracia. Es de prever que este horizonte se vaya ampliando todavía más en el futuro, no sin pausas ni retrocesos. De hecho, aquí nos hemos fijado en el modelo deliberativo de democracia practicado durante la fase concreta de las acampadas, pero otros conceptos o modelos de democracia han sido empleados por otros teóricos para explicar desarrollos posteriores del 15M: la contrademocracia (Pierre Rosanvallon), la democracia posrepresentativa (Simon Tormey), la democracia monitorizada (John Keane) o incluso planteamientos "tecnopolíticos". En este sentido, el contexto español ha sido "un campo de experimentación política continuada, donde la irrupción del movimiento $15 \mathrm{M}$ ha constituido un elemento desencadenante y catalizador para la innovación política (Feenstra et al., 2016, p. X). Pero 
es que, como se decía en la acampada de la Puerta del Sol: "vamos despacio porque vamos lejos". Los impactos de los movimientos sociales y los ciclos de protesta llevan tiempo. Por mucho que la opinión pública demande cambios súbitos y sustanciales tras las protestas (y juzgue que han fracasado si no los encuentra), sus resultados se sitúan más bien en el medio y largo plazo.

\section{NOTAS}

1. Este apartado incluye una ampliación y actualización de algunas de las ideas vertidas en Romanos (2011, 18 de noviembre).

\section{BIBLIOGRAFÍA}

Aron, R. (1973). Histoire et dialectique de la violence. París: Gallimard.

Boltanski, L. (2002). The left after May 1968 and the longing for total revolution. Thesis Eleven, 69, pp. 1-20. https://doi. org/10.1177/0725513602069001002

Calvo, K. (2013). Fighting for a Voice: The Spanish 15-M/Indignados Movement. En: Flesher Fominaya, C. y Cox, L. (eds.). Understanding European Movements: New Social Movements, Global Justice Struggles, Anti-Austerity Protest. Londres: Routledge, pp. 236-253.

Castells, M. (2012). Redes de indignación y esperanza. Madrid: Alianza.

Crowder, G. (1991). Classical anarchism: the political thought of Godwin, Proudhon, Bakunin and Kropotkin. Oxford: Clarendon Press.

De Jong, R. (1971). Provos and Kabouters. En: Apter, D. E. y Joll, J. (eds.). Anarchism Today. The MacMillan Press. https:// doi.org/10.1007/978-1-349-01074-5_9

Dryzek, J. S. (2000). Deliberative Democracy and Beyond. Nueva York: Oxford University Press.

Enzensberger, H. M. (1972). El corto verano de la anarquía. Vida y muerte de Durruti. Barcelona: Anagrama.

Feenstra, R. A., Tormey, S., Casero-Ripollés, A. y Keane, J. (2016). La reconfiguración de la democracia: el laboratorio político español. Granada: Comares.

Flesher Fominaya, C. (2014). Social Movements and Globalization: How Protests, Occupations and Uprisings are changing the World. Nueva York: Palgrave McMillan.
2. Así se indica en el manuscrito inédito de D. S. Patel (cedido por el autor) Roundabouts and Revolutions: Public Squares; Coordination, and the Diffusion of the Arab Uprisings.

Gerbaudo, P. (2012). Tweets and the Streets: Social Media and Contemporary Activism. Londres: Pluto Press.

Ginsborg, P., Passerini, L., Strath, B. y Wagner, P. (2002). 1968-2001: Measuring the Distance. Continuities and discontinuities in Recent History. Thesis Eleven, 68, pp. 5-10. https://doi. org/10.1177/0725513602068001001

Glasius, M. y Pleyers, G. (2013). The Global Moment of 2011: Democracy, Social Justice and Dignity. Development and Change, 44 (3), pp. 547-567. https:// doi.org/10.1111/dech.12034

Hadden, J. y Tarrow, S. (2007). Spillover or Spillout? The Global Justice Movement in the United States after 9/11. Mobilization, 12 (4), pp. 359-376.

Katsiaficas, G. N. (1987). The Imagination of the New Left: A Global Analysis of 1968. Boston: South End Press.

Kellner, D. (2005). Radical Politics, Marcuse, and the New Left. En: Kellner, D. (ed.). Collected Papers of Herbert Marcuse (vol. 3. The New Left and the 1960s). Londres: Routledge, pp. 1-37.

Kitschelt, H. (1993). Social Movements, Political Parties, and Democratic Theory. The Annals of the American Academy of Political and Social Sciences, 528, pp. 13-29. https://doi. org/10.1177/0002716293528001002

Klimke, M. y Scharloth, J. (2008). 1968 in Europe. A History of Protest and Activism, 1956-1977. Basingstoke: Palgrave MacMillan.

Kornetis, K. (2013). New Left and social movements in Europe. En: Snow, D. A., Porta, D. della, Klandermans, B. y McAdam, D. (eds). The Wiley-Blackwell En- cyclopedia of Social and Political Movements. Malden: Blackwell. https://doi. org/10.1002/9780470674871.wbespm142

Landwehr, C. (2009). Political Conflict and Political Preferences: Communicative Interaction Between Facts, Norms and Interests. Colchester: ECPR Press.

Lawrence, J. (2013). The international roots of the $99 \%$ and the "politics of anyone". IC- Revista Científica de Información y Comunicación, 10, pp. 53-72.

Lipovetsky, G. (1994). May '68, or the rise of transpolitical individualism. En: Lilla, M. (ed.). New French thought: political philosophy. Princeton: Princeton University Press, pp. 212-219. https://doi. org/10.1515/9781400863853.211

Maeckelbergh, M. (2011). Doing is Believing: Prefiguration as Strategic Practice in the Alterglobalization Movement. Social Movement Studies, 10 (1), pp. 1-20. https://doi.org/10.1080/14742837.201 1.545223

Mazower, M. (2000). Dark Continent. Europe's Twentieth Century. Nueva York: Vintage Books.

McDonough, T. (2002). Introduction: Ideology and the Situationist Utopia. En: McDonough, T. (ed.). Guy Debord and the Situationist International: Texts and Documents. Cambridge: The MIT Press.

Melucci, A. (1994). ¿Qué hay de nuevo en los nuevos movimientos sociales? En: Laraña, E. y Gusfield, J. (eds.). Los nuevos movimientos sociales: de la ideología a la identidad. Madrid: CIS, pp. 119-150.

Morland, D. (1997). Demanding the Impossible? Human Nature and Politics in 
Nineteenth-century Social Anarchism. Londres: Cassell.

Nehring, H. (2008). Great Britain. En: Klimke, M. y Scharloth, J. (eds.). 1968 in Europe. A History of Protest and Activism, 1956-1977. Basingstoke: Palgrave MacMillan, pp. 125-136. https://doi. org/10.1057/9780230611900_11

Nez, H. y Ganuza, E. (2012). Among Militants and Deliberative Laboratories: The "Indignados". En: Tejerina Montaña, B. y Perugorría, I. (eds). From social to political. New forms of mobilization and democratization (Conference proceedings). Bilbao: Universidad del País Vasco, pp. 119-136.

Pas, N. (2008). Subcultural movements. En: Klimke, M. y Scharloth, J. (eds.) (2008). 1968 in Europe. A History of Protest and Activism, 1956-1977. Basingstoke: Palgrave MacMillan, pp. 13-21. https://doi. org/10.1057/9780230611900_2

Perugorria, I. y Tejerina, B. (2018). Identity in the move: between emotional and relational processes of mobilization. En: Tejerina, B. y Perugorría, I. (eds.). Crisis and Social Mobilization in Contemporary Spain: The $15 \mathrm{M}$ Movement. Londres: Routledge, pp. 36-53.

Porta, D. della (2005a). Deliberation in Movement: Why and How to Study Deliberative Democracy and Social Movements. Acta Politica, 40, pp. 336350. https://doi.org/10.1057/palgrave. ap. 5500116

Porta, D. della (2005b). Making the Polis: Social Forums and Democracy in the Global Justice Movement, Mobilization, 10 (1), pp. 73-94.

Porta, D. della (2017). Global diffusion of protest: Riding the protest wave in the neoliberal crisis. Amsterdam: Amsterdam University Press. https:// doi.org/10.5117/9789462981690

Porta, D. della y Diani, M. (2011). Los movimientos sociales. Madrid: CIS-Universidad Complutense de Madrid.

Porta, D. della y Mattoni, A. (2014). Patterns of Diffusion and the Transnational
Dimension of Protest in the Movements of the Crisis: An Introduction. En: della Porta, D. y Mattoni, A. (eds.). Spreading Protest: Social Movements in Times of Crisis. Colchester: ECPR Press.

Porta, D. della y Mosca, L. (2007). In movimento: 'contamination' in action and the Italian Global Justice Movement. Global Networks, 7 (1), pp. 1-27. https://doi.org/10.1111/j.14710374.2006.00154.x

Porta, D. della y Reiter, H. (1998). Introduction: The Policing of Protest in Western Democracies. En: della Porta, D. y Reiter, H. (eds.). Policing Protest: The Control of Mass Demonstrations in Western Democracies. Minneapolis: University of Minnesota Press, pp. 1-32.

Rochon, T. (1998). Culture Moves: Ideas, Activism, and Changing Values. Princeton: Princeton University Press.

Romanos, E. (2007). Ideología libertaria y movilización clandestina. El anarquismo español durante el franquismo [Tesis doctoral inédita]. Florencia: Instituto Universitario Europeo.

Romanos, E. (2011, 18 de noviembre). El $15 \mathrm{M}$ y la democracia de los movimientos sociales. Dossier Débats autour du 15M. [En línea]. Disponible en http:// www.booksandideas.net/El-15M-y-lademocracia-de-los.htm

Romanos, E. (2013a). Collective Learning Processes within Social Movements: Some Insights into the Spanish 15M Movement. En: Flesher Fominaya, C. y Cox, L. (eds.). Understanding European Movements. Londres: Routledge, pp. 203-219.

Romanos, E. (2013b). Anarchism. En Snow, D. A., Porta, D. della, Klander mans, B. y McAdam, D. (eds.). The Wiley-Blackwell Encyclopedia of Social and Political Movements (vol. 1). Malden: Blackwell, pp. 53-56. https://doi. org/10.1002/9780470674871.wbespm009

Romanos, E. (2014). Evictions, Petitions and Escraches: Contentious Housing in Austerity Spain. Social Movement Studies,
13 (2), pp. 296-302. https://doi.org/10 $.1080 / 14742837.2013 .830567$

Romanos, E. (2016). De Tahrir a Wall Street por la Puerta del Sol: la difusión transnacional de los movimientos sociales en perspectiva comparada. Revista Española de Investigaciones Sociológicas, 154, pp. 103-118. https://doi. org/10.5477/cis/reis.154.103

Ross, K. (2002). May '68 and its afterlives. Chicago: University of Chicago Press. https://doi.org/10.7208/chicago/9780226728001.001.0001

Sampedro, V. y Lobera, J. (2014). The Spanish 15-M movement: a consensual dissent? Journal of Spanish Cultural Studies, 15 (1-2), pp. 61-80. https://doi.org/ 10.1080/14636204.2014.938466

Sanbonmatsu, J. (2004). The postmodern prince: critical theory, left strategy, and the making of a new political subject. Nueva York: Monthly Review Press.

Seidman, M. (2004). The Imaginary Revolution. Parisian Students and Workers in 1968. Nueva York: Berghahn. https:// doi.org/10.2307/j.ctt1x76fq7

Talpin, J. (2011). Schools of Democracy. How Ordinary Citizens (Sometimes) Become Competent in Participatory Budgeting Institutions. Colchester: ECPR Press.

Tarrow, S. (1994). Power in Movement. Social Movements, Collective Action and Politics. Cambridge: Cambridge University Press.

Tejerina, B., Perugorria, I., Benski, T. y Langman, L. (2013). From indignation to occupation: A new wave of global mobilization. Current Sociology, 61 (4), pp. 377-392. https://doi. org/10.1177/0011392113479738

Tilly, C. (1986). The Contentious French. Cambridge, MA: Harvard University Press. https://doi.org/10.4159/harvard.9780674433984

Yack, B. (1986). The longing for total revolution. Philosophic sources of social discontent from Rousseau to Marx and Nietzsche. Princeton: Princeton University Press. 